\title{
Shape Optimization of the Air Compressor Supporting Leg by Using the Optimization Technique
}

\author{
Witchakorn Wongchanasit ${ }^{1, a}$, Teetut Dolwichai ${ }^{1, b}$ and Chalothorn Thumthae ${ }^{1}$ \\ ${ }^{1}$ School of Mechanical Engineering, Institute of Engineering, \\ Suranaree University of Technology 30000, Thailand \\ aw_witchakorn@hotmail.co.th, ${ }^{\mathrm{b}}$ prapun@sut.ac.th
}

\begin{abstract}
Keywords: Air compressor supporting leg, Optimization technique, Genetic algorithms, Shape
\end{abstract} optimization, Materials reduction.

\begin{abstract}
The air conditioner installation in general, the air compressor is installed at the external of the building that has well for heat rejected and pretty location. The air compressor must be used the supporting leg for support the its weight. Now a day, the production of supporting leg has the measure to reduce its cost by reduction of material weight but its strength usable. According to material weight reduction, supporting leg shape and cross section may be change. This is the point of this work. The objective work is minimizing the material weight but it also supportable its weight. This problem can solve by the optimization technique. The optimization technique which this way is known and the most widely used for structural design problem is Genetic Algorithms. This research used the NonDominated Sorting Genetic Algorithms method for solve the multi-objective problem which it are minimize cross sectional area and minimize deflection of supporting leg and the constraint conditions is the supporting leg's bending stress does not exceed the strength of materials.
\end{abstract}

\section{Introduction}

In the present, Air conditioning is important and appreciated worldwide. Because the human needs comfortable temperature for daily routine. The air conditioner installation depending on several factors such as the width of installation area. The solution of the above mentioned is the air compressor is installed at the external of the building. The device which is important to installation is the air compressor supporting leg. As shown in Fig. 1.

In this research we will consider about a reduction of the materials of the supporting leg. Such considerations, which may affect the shape and cross-sectional area. The engineering knowledge that can be applied to this problem is the optimization technique, which must be considered in constraints of problem such as the bending force on the supporting leg, conversion of the cross sectional area, included to precision of the optimal shape after the calculation.

From the Previous paragraph, we must be used the knowledge and techniques of the optimization in the research. Evolutionary algorithms is one way of the optimization technique which the most widely used. It is Genetic algorithms. Genetic algorithm was developed based on the principles of evolution and natural selection and the most widely used in engineering such as electrical work, structural work included the pipe system. This methodology have advantages are it is easy to used, didn't calculate the derivative of the solution, chance of error is very little, and most importantly, this way be applied to the design possibilities., [1]. If the genetic algorithm applied to solve the optimal shape of the air compressor supporting leg it would be useful to solve of this research.

To solving the problem of the air compressor supporting leg. It used the same calculations with the calculations of the cantilever beam. From the studying about the research of cantilever beam have information that genetic algorithms was used to calculate the most about the calculations of the beam structure. Moreover, it have been applied to the calculation with other techniques such as genetic algorithms with Constructive geometry topology optimization method, [2], with 1-0 binary variables techniques, [6] with Boundary element method, [7], with Boundary element method and Free-form deformation technique, [8]. Moreover, genetic algorithms can also be calculated with a different 
structure such as Hat-Shaped Cold-Formed, [5]. The calculation result of each research above they get the reliable results.

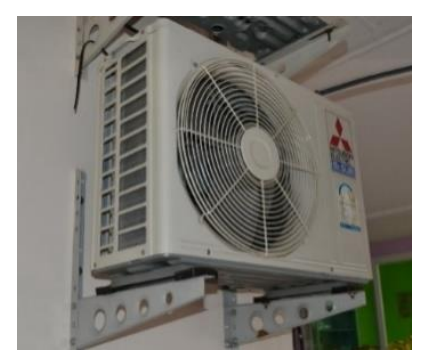

Figure 1. the air compressor unit and the supporting leg.

\section{Stresses and Deflections of the Air Compressor Supporting Leg}

Load on the supporting leg. Load and support conditions on the supporting leg is shown in Fig. 2. Consideration from Fig. 2. (a) we can know that the weight of air compressor unit is the distributed load on the supporting leg and fixed support.

Design variables of $\mathbf{C}$-section. For this work we chosen the $\mathrm{C}$-section because the $\mathrm{C}$-section can be reduced the materials more than the other cross section, [4]. When they compared the results of calculation between $\mathrm{C}$-section, I-section and T-section. The design variables is shown in Fig. 2 (b). The design elements are calculated as follows.

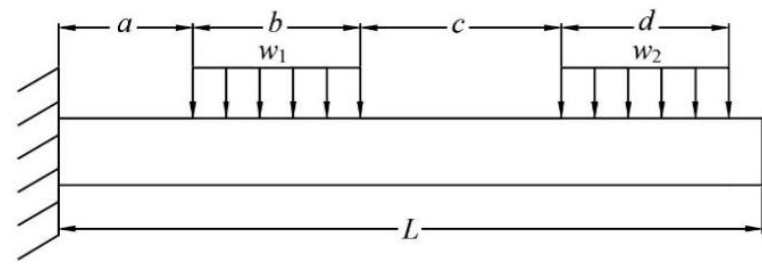

(a)

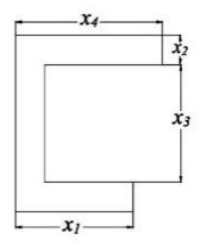

(b)

Figure 2. (a) Loads on the supporting leg and (b) design variables of C-section

\section{Optimization Technique}

Genetic Algorithms. (GA) was developed based on the principles of evolution and natural selection. A population of solutions (genes) is generated and then the next generation is produced by mating pairs of these genes. The search procedure starts with an initial population along with their respective fitness values. Three main operations, selection, crossover and mutation, are then carried out in order to generate the next population. The population is evolved repeatedly until an optimum is achieved.

This operation is presented for single objective function which this approach may finding the optimal solution for some problem but other problem need the objective function is more than one function, for example, The structure is designed for the minimum weight. Meanwhile, it also needs to be maximum strength. These problem is called Multi-objective optimization.

Multi-objective optimization. Mathematically problems for multi-objective optimization is expressed as :

$$
\mathrm{f}=\left\{f_{1}(\mathrm{x}), \ldots, f_{\mathrm{p}}(\mathrm{x})\right\}
$$

The constraint conditions are expressed as :

$$
\begin{aligned}
& g_{\mathrm{i}}(\mathrm{x}) \leq 0 \\
& h_{\mathrm{i}}(\mathrm{x})=0
\end{aligned}
$$


where $\mathrm{x}$ is the design variables, $f_{\mathrm{i}}$ is the objective function, $g_{\mathrm{i}}$ is the constraint conditions for inequality form and $h_{\mathrm{i}}$ is the constraint conditions for equality form.

From Fig. 3, it shown plotting of the solutions on the objective function domain or the feasible solution which it corresponds to the constraint conditions. So the optimal solutions for bi-objective optimization problem will be in the feasible region. the minimum of bi-objective function which the solutions is at the edge of the feasible region and it's on the curve between $\min f_{1}$ and $\min f_{2}$ point. These solutions called Pareto frontier. All point on pareto frontier are the optimal solutions for the minimum optimization problem and we can select the solutions on pareto frontier depending on our requirements. Pareto frontier can be obtained by non-dominated technique.

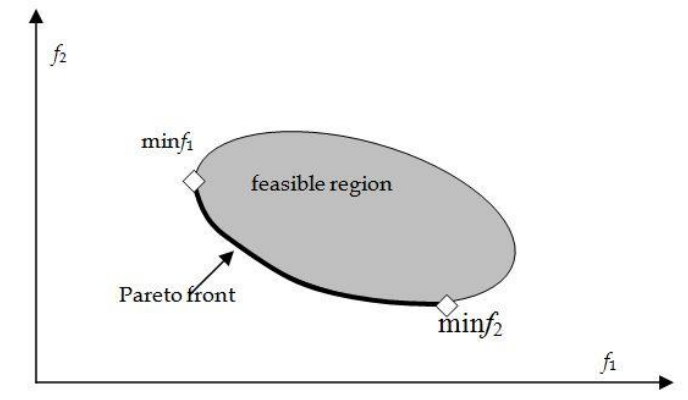

Figure 3. Pareto frontier for bi-objective optimization problem

Non-dominated sorting genetic algorithms II. (NSGAII) is the most widely used in engineering work for multi-objective problem. It's developed the single objective of GA to the multi-objective in order to it can find the better solutions. NSGAII procedure is shown in Fig. 4.

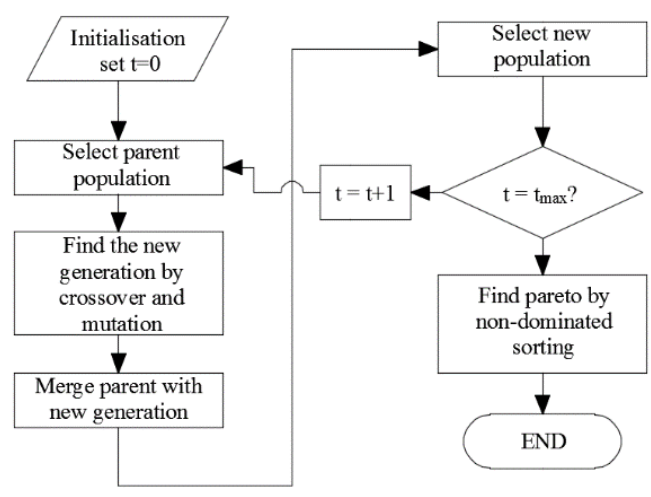

Figure 4. Flow chart for NSGAII

\section{Objective functions and Constraint conditions}

The objective of the problem is to find the optimum dimensions of the supporting leg, such that the cross section area and the deflection are both minimized and the constraint conditions is the supporting leg's bending stress does not exceed the strength of materials,[3]. Objective function as :

$$
F\left(X^{*}\right)=\left[f_{1}\left(X^{*}\right), f_{2}\left(X^{*}\right)\right]
$$

Where $X^{*}$ is the optimum design variables, $f_{1}(X)$ is the objective function of the area and $f_{2}(X)$ is the objective function of the deflection.

Objective function above mentioned can be expressed as :

$$
\begin{aligned}
& \min f_{1}(X)=\min A(x) \\
& \min f_{2}(X)=\min \delta(x)
\end{aligned}
$$


the constraint conditions is expressed as :

$$
g(x)=\sigma_{\max } \leq \sigma_{\text {allow }}(x)
$$

Where $A$ is the cross section area, $\delta$ is the static deflection, $\sigma_{\max }$ is the maximum stress and $\sigma_{\text {allow }}$ is allowable stress.

We defined the variables follows. The values of length $a=8 \mathrm{~cm} ., b=10 \mathrm{~cm}$. , $c=16 \mathrm{~cm}$.,$d=10$ $\mathrm{cm}$. , $L=45 \mathrm{~cm}$. The values of forces $w_{1}=3000 \mathrm{~N} / \mathrm{m}, w_{2}=3000 \mathrm{~N} / \mathrm{m}$, Young's modulus E $=200$ $\mathrm{GPa}$, the centroid point of area and the moment of inertia can calculated from Eq. 9 and Eq. 10, respectively.

$$
\begin{aligned}
& c=\frac{x_{2}\left(x_{1}+2 x_{3}+3 x_{4}\right)+x_{3}^{2}+2 x_{3} x_{4}}{2\left(x_{1}+x_{3}+x_{4}\right)} \\
& I=\frac{x_{2}}{12}\left[\left(x_{1} x_{2}^{2}+x_{3}^{3}+x_{2}^{2} x_{4}\right)+6\left(x_{1}\left(2 \bar{y}-x_{2}\right)+x_{3}\left(2 \bar{y}-x_{3}-2 x_{2}\right)+x_{4}\left(3 x_{2}+2 x_{3}-2 \bar{y}\right)\right)\right]
\end{aligned}
$$

The constraints are

$$
0.005 \leq x_{1} \leq 0.03,0.0005 \leq x_{2} \leq 0.002,0.09 \leq x_{3} \leq 0.119 \text { and } 0.015 \leq x_{4} \leq 0.03
$$

The objective function of cross sectional area and deflection are

$$
\begin{aligned}
& A=f_{1}(X)=x_{2}\left(x_{1}+x_{3}+x_{4}\right) \\
& \delta=f_{2}(X)=\frac{5.401(10)^{-11}}{I}
\end{aligned}
$$

Subject to the bending stress constraint

$$
\sigma=g(x)=\frac{156 c}{I} \leq 250\left(10^{9}\right)
$$

\section{Result and Discussion}

Fig. 5. illustrates the results of calculation which Fig. 5 (a) shown the optimal point on the Pareto frontier of this problem and Fig. 5 (b) shown the cross sectional area of each point on Pareto frontier which points are close a y-axis affect to a small cross section area and it gets the large values deflection. On the other hand, when points are close a $\mathrm{x}$-axis affect to a large values of cross section

\begin{tabular}{|c|c|c|c|c|c|c|}
\hline \multirow{2}{*}{$\begin{array}{l}\text { Optimal } \\
\text { point }\end{array}$} & \multicolumn{4}{|c|}{ Design variables (m) } & \multirow{2}{*}{$\begin{array}{c}\text { Cross sectional } \\
\text { area }\left(\mathrm{m}^{2}\right)\end{array}$} & \multirow{2}{*}{$\begin{array}{l}\text { Deflection } \\
\text { (m) }\end{array}$} \\
\hline & $x_{1}$ & $x_{2}$ & $x_{3}$ & $x_{4}$ & & \\
\hline 1 & 0.0051 & $5.0075\left(10^{-4}\right)$ & 0.0901 & 0.0152 & $5.5318\left(10^{-5}\right)$ & 0.0018 \\
\hline 2 & 0.0052 & $5.0108\left(10^{-4}\right)$ & 0.0943 & 0.0153 & $5.7496\left(10^{-5}\right)$ & 0.0015 \\
\hline 3 & 0.0051 & $5.0075\left(10^{-4}\right)$ & 0.1060 & 0.0152 & $6.3254\left(10^{-5}\right)$ & 0.0011 \\
\hline 4 & 0.0207 & 0.0014 & 0.1011 & 0.0184 & $1.9067\left(10^{-4}\right)$ & $4.6149\left(10^{-4}\right)$ \\
\hline 5 & 0.0067 & 0.0019 & 0.1055 & 0.0183 & $2.8784\left(10^{-4}\right)$ & $2.9102\left(10^{-4}\right)$ \\
\hline 6 & 0.0253 & 0.0018 & 0.1169 & 0.0264 & $3.0161\left(10^{-4}\right)$ & $2.2691\left(10^{-4}\right)$ \\
\hline
\end{tabular}
area and it gets the small values deflection. The values of design variables shown in table 1. point 1 reduces the cross sectional area from point 6 about $18.34 \%$ but the deflection increase depending on the number of each point which point 6 can allow loads from the compressor unit better than smaller optimal point because the deflection values are the smallest but it used the most materials. So we can choose each optimal point depending on the requirement.

Table 1. The values of each point on Pareto frontier 


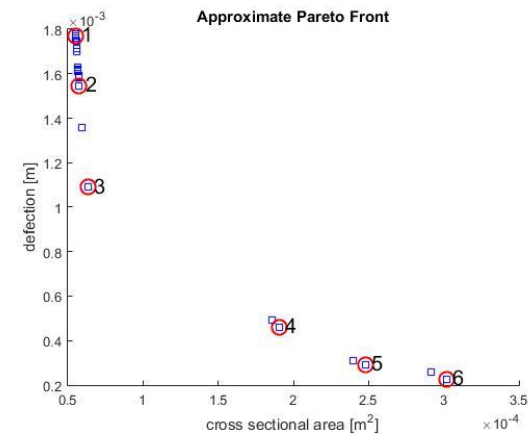

(a)
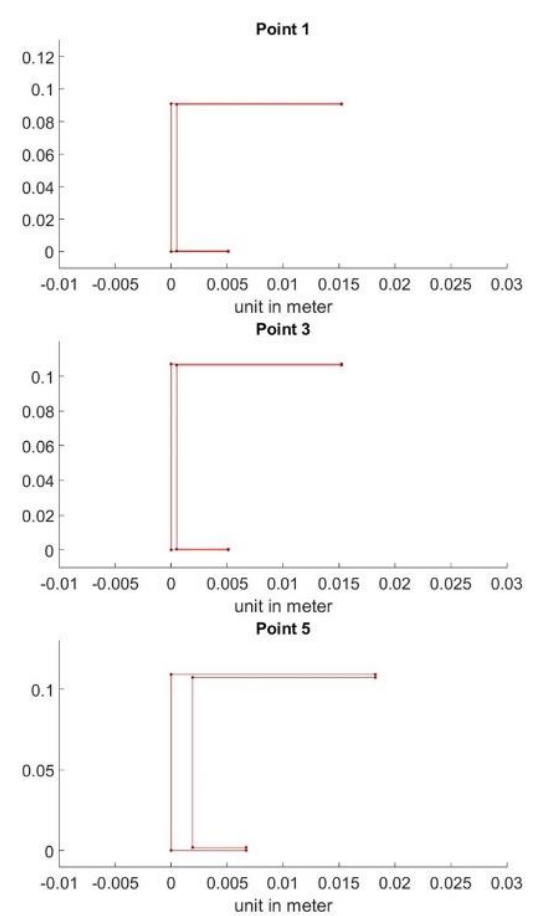

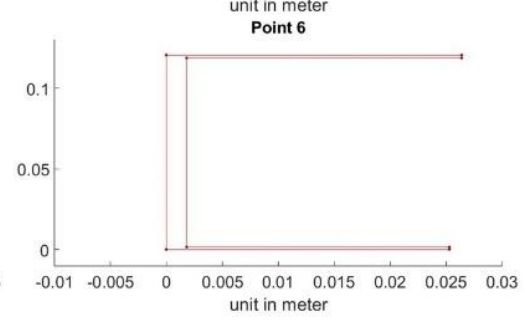

(b)
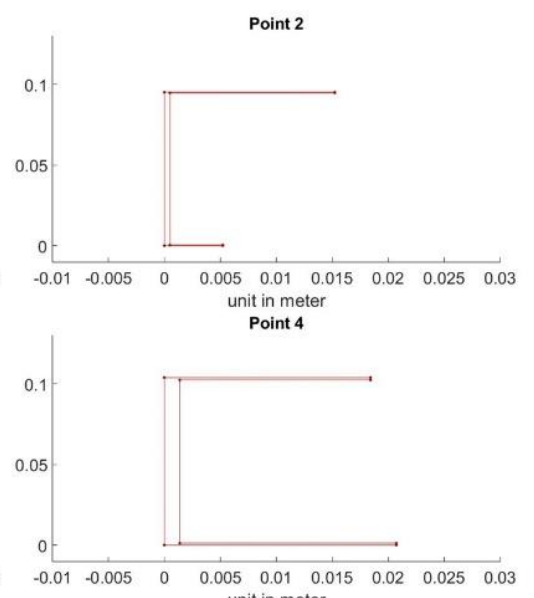

Figure 5. Pareto frontier and cross-sectional area

\section{Conclusion}

The optimal points shown on the Pareto frontier which all points are the optimal solutions. It indicated the optimal values of the cross sectional area and the deflection of the supporting leg. We can choose each point depending on the requirement of work and usability. This research used NSGAII for the calculations which it can made a good results and high precision follows the constraint conditions. Moreover, this method can apply to solve the other structural design problem and more complicated problem in the future.

\section{References}

[1] S. Bureerat, Optimization of Mechanical Engineering systems , Khonkean, Thailand, 2013.

[2] F. Ahmed, B. Bhattacharya, K. Deb., Constructive Solid Geometry Based Topology Optimization using Evolutionary Algorithms, KanGAL Report Number 2012013 (2012).

[3] A. Khazaee, H.M. Naimi, Two Multi-Objective Genetic Algorithms for Finding Opimum Design of an I-Beam, Scientific Research 3 (2011) 1054-1061.

[4] F.M. Nadela, J.E.C. Lope, Comparative Strength of Common Structural Shapes Using Genetic Algorithms, Proceeding of World Congress on Engineering 2009 Vol II (2007).

[5] W. Lu, P. Mäkeläinen, Augmented Lagriangian Genetic Algorithms for Optimal Design of HatShaped Cold-Formed, Building Materials Structures and Techniques, BMB2007 (2007).

[6] S. Bureerat, J. Limtragool, Performance Enhancement of Search for Structural Topology Optimization, Finite Element in Analysis and Design 42 (2006) 547-566.

[7] T. Burczynski, G. Kokot, Evolutionary Algorithms and Boundary Element Method in Generalized Shape Optimization, JOURNAL OF THEORETICAL AND APPLIED MECHANICS 412 (2003) 341-364.

[8] E. Kita, H. Tanie, Shape Optimization of Continuum Structures by Genetic Algorithms and Boundary Element Method, Engineering Analysis with Boundary Element 19 (1997) 129-136. 